\title{
Atiyah sequences, connections and characteristic forms for principal bundles over groupoids and stacks
}

\author{
Indranil Biswas ${ }^{\mathrm{a}}$, Frank Neumann ${ }^{\mathrm{b}}$ \\ ${ }^{a}$ School of Mathematics, Tata Institute of Fundamental Research, Homi Bhabha Road, Mumbai 400005, India \\ b Department of Mathematics, University of Leicester, University Road, Leicester LE1 7RH, United Kingdom
}

\begin{abstract}
We construct connections and characteristic forms for principal bundles over groupoids and stacks in the differentiable, holomorphic and algebraic categories using Atiyah exact sequences associated to transversal tangential distributions.

Résumé

Suites d'Atiyah, connexions et formes caractéristiques pour les fibrés principaux sur les groupoïdes et les champs. Nous construisons les connexions et formes caractéristiques pour les fibrés principaux sur les groupoïdes et les champs dans les catégorie différentiables, holomorphes et algébriques à l'aide des suites d'Atiyah associées aux distributions transversales tangentielles.
\end{abstract}

\section{Version française abrégée}

Dans cette note, nous commenons à développer une théorie générale des connexions et des classes caractéristiques pour les fibrés $G$-principaux sur les groupoïdes et les champs. Les constructions présentées ici fonctionnent aussi bien dans les catégories différentiables, holomorphes et algébriques et pour les groupoïdes qui ne sont pas nécessairement étales. Etant donné un groupoïde $\mathbb{X}:=\left[X_{1} \rightrightarrows X_{0}\right]$ de telle sorte que la source $s$ soit une submersion, nous définissons une connexion comme une distribution particulière transversale $\mathcal{H} \subset T X_{1}$ dans l'espace tangent $T X_{1}$ (voir Définition 3.1).

À l'aide de présentations par des groupoïdes il est aussi possible de cette manière de définir les connexions sur les champs. Pour le cas particulier des champs de Deligne-Mumford, qui sont présentés par les groupoïdes, étales, les connexions existent toujours.

Etant donné un groupe de Lie $G$ et un $G$-fibré principal sur un groupoïde $\mathbb{X}:=\left[X_{1} \rightrightarrows X_{0}\right]$ avec une connexion $\mathcal{H}$ nous contruisons une connexion induite $\widetilde{\mathcal{H}}$ pour le $G$-fibré principal. Un tel fibré est donné par un $G$-fibré principal $\alpha: E_{G} \rightarrow X_{0}$ sur l'espace $X_{0}$ et nous prouvons que l'existence d'une connexion correspond à l'existence d'une section de la suite d'Atiyah associée [1]

Email addresses: indranil@math.tifr.res.in (Indranil Biswas), fn8@mcs.le.ac.uk (Frank Neumann). 


$$
0 \rightarrow \operatorname{ad}\left(E_{G}\right) \rightarrow \operatorname{At}\left(E_{G}\right) \rightarrow T X_{0} \rightarrow 0 .
$$

En utilisant les distributions $\mathcal{H}$ transversalles tangentielles nous construisons aussi des formes de courbure et des autres formes caractéristiques différentielles pour les $G$-fibrés principaux sur les groupoïdes. Dans le cas des champs Deligne-Mumford cette théorie correspond aux constructions de [4].

\section{Introduction}

In this note we outline a general theory of connections and characteristic classes for principal $G$-bundles over groupoids and stacks, which works equally well in the differentiable, holomorphic and algebraic setting and for groupoids, which are not necessarily étale. Given a groupoid $\mathbb{X}:=\left[X_{1} \rightrightarrows X_{0}\right]$ in one of these three settings with both $X_{0}$ and $X_{1}$ smooth and the source map $s$ being a submersion, we define a connection on it as a distribution $\mathcal{H} \subset T X_{1}$ transversal to the fibers of $s$. It is said to be flat if $\mathcal{H}$ is integrable. Using groupoid presentations this gives rise to connections and flat connections on stacks under certain compatibility conditions. For the particular case of Deligne-Mumford stacks presented by étale groupoids a connection always exists, because in this case we have $\mathcal{H}=T X_{1}$.

Given a Lie group $G$ and a principal $G$-bundle over a groupoid $\mathbb{X}:=\left[X_{1} \rightrightarrows X_{0}\right]$ with connection $\mathcal{H}$, we can define a connection for the principal $G$-bundle. A $G$-bundle over the groupoid $\mathbb{X}$ is basically given by a principal $G$-bundle $\alpha: E_{G} \rightarrow X_{0}$ and some extra data as explained in Section 2. A connection for the principal $G$-bundle over the groupoid $\mathbb{X}$ corresponds to a splitting of the associated Atiyah sequence [1]

$$
0 \rightarrow \operatorname{ad}\left(E_{G}\right) \rightarrow \operatorname{At}\left(E_{G}\right) \rightarrow T X_{0} \rightarrow 0
$$

which is compatible with the data (see Section 4). This allows us to define curvature and characteristic differential forms. Using adequate groupoid presentations this gives a general framework for studying connections and characteristic forms for principal $G$-bundles over stacks. In the case of Deligne-Mumford stacks this corresponds to the constructions in [4].

Full details of the results presented here will appear in [5]. There we also discuss relations of our approach with Behrend's theory of cofoliations on stacks [2], the various approaches to Chern-Weil theory for étale groupoids via simplicial manifolds $[6,9,15]$ and the infinitesimal theory via Lie algebroids $[8$, 16]. Another aspect of [5] is the systematic construction of secondary characteristic classes and Deligne cohomology for principal $G$-bundles with connections over general groupoids and stacks. This extends the theory of secondary characteristic classes of Cheeger-Simons differential characters to stacks and general groupoids, which for differentiable manifolds were originally constructed in [7], for algebraic varieties in [10] and recently in the case of étale groupoids in [11].

\section{Groupoids, stacks and principal bundles}

We will consider groupoids and stacks over the following three categories: the differentiable category of $\mathcal{C}^{\infty}$-manifolds, the holomorphic category of complex analytic manifolds and the algebraic category of smooth schemes of finite type over a field $k$ of characteristic zero. We will refer to any of these categories as the category $\mathfrak{S}$ of smooth spaces. Any smooth space $X$ has a structure sheaf $\mathcal{O}_{X}$. Vector bundles are identified with their locally free sheaves of $\mathcal{O}_{X}$-modules. The tangent bundle of $X$ will be denoted by $T X$.

A groupoid $\mathbb{X}:=\left[X_{1} \rightrightarrows X_{0}\right]$ will mean a groupoid internal to the category $\mathfrak{S}$ of smooth spaces, i.e., the space $X_{1}$ of arrows and the space $X_{0}$ of objects are objects of $\mathfrak{S}$ and all structure morphisms

$$
m: X_{1} \times_{X_{0}} X_{1} \rightarrow X_{1}, s, t: X_{1} \rightarrow X_{0}, i: X_{1} \rightarrow X_{1}, e: X_{0} \rightarrow X_{1}
$$


are morphisms in $\mathfrak{S}$ and the source map $s$ is a submersion. Here $t$ is the target map, $m$ denotes the multiplication map, $e$ the identity section and $i$ the inversion map of the groupoid. If in addition $s$ and $t$ are étale, the groupoid $\mathbb{X}=\left[X_{1} \rightrightarrows X_{0}\right]$ is called étale. If in addition, the anchor map

$$
(s, t): X_{1} \rightarrow X_{0} \times X_{0}
$$

is proper, the groupoid is called a proper groupoid.

Every groupoid $\mathbb{X}=\left[X_{1} \rightrightarrows X_{0}\right]$ has an induced tangent groupoid $T \mathbb{X}:=\left[T X_{1} \rightrightarrows T X_{0}\right]$. A Lie group $G$ is a groupoid $[G \rightrightarrows *]$ with one object, i.e., the space $X_{0}$ is just a point.

A stack $\mathfrak{X}$ will mean a stack over the category $\mathfrak{S}$ (endowed with the "submersion" Grothendieck topology) associated to a groupoid $\left[X_{1} \rightrightarrows X_{0}\right]$, such that $\mathfrak{X}$ has a presentation or atlas $X_{1} \rightrightarrows X_{0} \rightarrow \mathfrak{X}$, which is a representable surjective submersion. A stack $\mathfrak{X}$ is called a Deligne-Mumford stack if there is a presentation $X_{1} \rightrightarrows X_{0} \rightarrow \mathfrak{X}$ such that the groupoid $\left[X_{1} \rightrightarrows X_{0}\right]$ is étale. Orbifolds correspond to proper Deligne-Mumford stacks (see [2,13]). Different presentations of the same stack are given by Morita equivalent groupoids and stacks can be seen as Morita equivalence classes of groupoids [3]. Given a groupoid $\mathbb{X}$, the associated category $\mathfrak{B} \mathbb{X}$ of $\mathbb{X}$-torsors is a stack $[3,5]$.

Definition 2.1 Let $\mathbb{X}=\left[X_{1} \rightrightarrows X_{0}\right]$ be a groupoid. $A \mathbb{X}$-space is given by an object $P$ of $\mathfrak{S}$ together with a smooth map $\pi: P \rightarrow X_{0}$ and a map $\sigma: Q \rightarrow P, \sigma(\gamma, x):=\gamma \cdot x$, where $Q:=X_{1} \times_{s, X_{0}, \pi} P$ is the fiber product such that:

(i) For all $x \in P$, we have: $e(\pi(x)) \cdot x=x$

(ii) For all $x \in P$ and all $\gamma, \delta \in X_{1}$ such that $\pi(x)=s(\gamma)$ and $t(\gamma)=s(\delta)$ we have: $(\delta \cdot \gamma) \cdot x=\delta \cdot(\gamma \cdot x)$. Let $G$ be a Lie group and $\mathbb{X}=\left[X_{1} \rightrightarrows X_{0}\right]$ a groupoid. A principal $G$-bundle over $\mathbb{X}$, denoted by $\left[s^{*} E_{G} \rightrightarrows\right.$ $\left.E_{G}\right]$ is a principal right $G$-bundle $\pi: E_{G} \rightarrow X_{0}$, which is also a $\mathbb{X}$-space such that for all $x \in E_{G}$ and all $\gamma \in X_{1}$ with $s(\gamma)=\pi(x)$ we have $(\gamma \cdot x) \cdot g=\gamma \cdot(x \cdot g)$ for all $g \in G$.

It can be shown that if $\mathbb{X}=\left[X_{1} \rightrightarrows X_{0}\right]$ and $\mathbb{Y}=\left[Y_{1} \rightrightarrows Y_{0}\right]$ are Morita equivalent groupoids, then there is an equivalence between their categories of principal $G$-bundles (see $[3,15]$ ).

Principal $G$-bundles over a stack $\mathfrak{X}$ can be defined directly using a presentation. A principal $G$-bundle $\mathfrak{E}_{G}$ over a stack $\mathfrak{X}$ is then given by a principal $G$-bundle $E_{G} \rightarrow X_{0}$ for an atlas $X_{0} \rightarrow \mathfrak{X}$ together with an isomorphism of the pullbacks $p_{1}^{*} E_{G} \stackrel{\sim}{\rightarrow} p_{2}^{*} E_{G}$ on the fiber product $X_{0} \times \mathfrak{X} X_{0}$ satisfying the cocycle condition on $X_{0} \times_{\mathfrak{X}} X_{0} \times_{\mathfrak{X}} X_{0}$. It turns out that for any submersion $f: U \rightarrow \mathfrak{X}$ this datum defines a principal $G$-bundle $E_{G} \rightarrow U$ over $U$, because $X_{0} \times_{\mathfrak{X}} U \rightarrow U$ has local sections. Therefore we get a stack $\mathfrak{E}_{G}$ and the $G$-multiplication map glues and there is a natural morphism $G \times \mathfrak{E}_{G} \rightarrow \mathfrak{E}_{G}($ see $[4,12])$. Similarly, we can define vector bundles over a groupoid $\mathbb{X}$ and vector bundles over a stack $\mathfrak{X}$. They can be identified with respective principal $\mathrm{GL}_{n}$-bundles.

Let $G$ be a Lie group, $\mathfrak{X}$ a stack and $\mathbb{X}=\left[X_{1} \rightrightarrows X_{0}\right]$ a groupoid presenting $\mathfrak{X}$. Then there is a canonical equivalence between the category of principal $G$-bundles over $\mathfrak{X}$ and the category of principal $G$-bundles over $\mathbb{X}($ see $[3,12])$.

\section{Connections on groupoids and stacks}

Let $\left(\mathbb{X}=\left[X_{1} \rightrightarrows X_{0}\right], s, t, m, e, i\right)$ be a groupoid. The source map $s: X_{1} \rightarrow X_{0}$ is a submersion and therefore the differential $d s: T X_{1} \rightarrow s^{*} T X_{0}$ of $s$ is surjective. Let

$$
\mathscr{K}:=\operatorname{kernel}(d s) \subset T X_{1}
$$

be the vertical tangent bundle for $s$. Fix a distribution given by a subbundle $\mathscr{H} \subset T X_{1}$ such that the natural map $\mathscr{K} \oplus \mathscr{H} \rightarrow T X_{1}$ is an isomorphism. Define

$$
d_{\mathscr{H}} s:=\left.(d s)\right|_{\mathscr{H}}: \mathscr{H} \rightarrow s^{*} T X_{0}
$$


to be the restriction of $d s$ to $\mathscr{H}$. Note that $d_{\mathscr{H}} s$ is an isomorphism.

Let $d t: T X_{1} \rightarrow t^{*} T X_{0}$ be the differential of $t$. We get a map

$$
\theta:=d t \circ\left(d_{\mathscr{H}} s\right)^{-1}: s^{*} T X_{0} \rightarrow t^{*} T X_{0} .
$$

For any $y \in X_{1}$, let

$$
\theta_{y}:=\left.\theta\right|_{\left(s^{*} T X_{0}\right)_{y}}: T_{s(y)} X_{0} \rightarrow T_{t(y)} X_{0}
$$

be the restriction of $\theta$ to the fiber $\left(s^{*} T X_{0}\right)_{y}=T_{s(y)} X_{0}$. We now define:

Definition $3.1 A$ connection on a groupoid $\mathbb{X}=\left[X_{1} \rightrightarrows X_{0}\right]$ is a distribution $\mathscr{H} \subset T X_{1}$ such that:

(i) for every $x \in X_{0}$, the image of the differential de $(x): T_{x} X_{0} \rightarrow T_{e(x)} X_{1}$ coincides with the subspace $\mathscr{H}_{e(x)} \subset T_{e(x)} X_{1}$, and

(ii) for every $y, z \in X_{1}$ with $t(y)=s(z)$, the homomorphism

$$
\theta_{m(z, y)}: T_{s(y)} X_{0} \rightarrow T_{t(z)} X_{0}
$$

coincides with the composition $\theta_{z} \circ \theta_{y}$.

A connection on $\mathbb{X}$ is said to be flat if the distribution $\mathscr{H}$ is integrable.

Remark 3.2 The above definition of (flat) connections on a groupoid is equivalent to the one given by Behrend in [2] and the one given by Laurent-Gengoux, Tu and Xu [15] in the case of étale groupoids. Flat connections in the differentiable setting were also introduced independently as étalifications by Tang [17]. In fact, a connection as defined above gives a subgroupoid of the tangent groupoid $T \mathbb{X}$, which in the differentiable category is equivalent to the horizontal paths forming a subgroupoid of the path groupoid of $\mathbb{X}$. This definition is also used by Laurent-Gengoux, Stiénon and Xu in [14] to define connections for the general framework of non-abelian differentiable gerbes via Ehresmann connections on Lie groupoid extensions.

Let $\mathscr{H}$ be a connection on a groupoid $\mathbb{X}=\left[X_{1} \rightrightarrows X_{0}\right]$ and $\tau: T X_{0} \rightarrow X_{0}$ be the natural projection. Consider the fiber product $Y_{1}:=X_{1} \times_{s, X_{0}, \tau} T X_{0}$. Let $s^{\prime}: Y_{1} \rightarrow T X_{0}$ be the projection to the second factor and let $t^{\prime}: Y_{1} \rightarrow T X_{0}$ be the morphism defined by $t^{\prime}(x, v)=\theta_{x}(v) \in T_{t(x)} X_{0}$, where $\theta_{x}$ is defined as in (2). Furthermore, let $p: Y_{1}=X_{1} \times_{s, X_{0}, \tau} T X_{0} \rightarrow X_{1}$ be the projection to the first factor. For any $z, y \in Y_{1}$ with $t^{\prime}(y)=s^{\prime}(z)$, define $m^{\prime}(z, y):=\left(m(p(z), p(y)), s^{\prime}(y)\right)$. Let $e^{\prime}: T X_{0} \rightarrow Y_{1}$ be the morphism defined by $v \mapsto(e(f(v)), v)$ and finally let $i^{\prime}: Y_{1} \rightarrow Y_{1}$ be the involution defined by $(z, v) \mapsto\left(i(z), \theta_{z}(v)\right)$. We note that $\left(\left[Y_{1} \rightrightarrows T X_{0}\right], s^{\prime}, t^{\prime}, m^{\prime}, e^{\prime}, i^{\prime}\right)$ is a groupoid. In other words, $T X_{0}$ becomes a vector bundle over the groupoid $\mathbb{X}$.

(Flat) connections on groupoids as defined above behave well with respect to pullbacks of cartesian morphisms and Morita equivalences of groupoids and therefore we can speak of (flat) connections on stacks. A (flat) connection on a groupoid $\mathbb{X}$ descends to the stack $\mathfrak{B} \mathbb{X}$ of $\mathbb{X}$-torsors [5].

However, even in the differentiable category these connections might not always exist. But if $\mathbb{X}$ is an étale groupoid there is always a connection on $\mathbb{X}$, because then we have $\mathcal{H}=T X_{1}$ and so get actually a unique flat connection (see also [2]). Therefore connections on a Deligne-Mumford stack $\mathfrak{X}$ always exist and can be defined using an étale groupoid presentation.

\section{Connections on principal bundles over groupoids and stacks}

Let $G$ be a Lie group and let $\alpha: E_{G} \rightarrow X_{0}$ be a principal $G$-bundle over $X_{0}$. The adjoint vector bundle for $E_{G}$ will be denoted by $\operatorname{ad}\left(E_{G}\right)$. Let $\operatorname{At}\left(E_{G}\right):=\left(\alpha_{*} T E_{G}\right)^{G}$ be the Atiyah bundle for $E_{G}$ [1]. We have the exact sequence of Atiyah [1]:

$$
0 \rightarrow \operatorname{ad}\left(E_{G}\right) \rightarrow \operatorname{At}\left(E_{G}\right) \rightarrow T X_{0} \rightarrow 0 .
$$


The projection $\operatorname{At}\left(E_{G}\right) \rightarrow T X_{0}$ is given by the differential $d \alpha$. We recall that a connection on $E_{G}$ is a splitting of the exact sequence (3) (see [1]).

Fix a connection $\mathscr{H}$ on the groupoid $\mathbb{X}=\left[X_{1} \rightrightarrows X_{0}\right]$ and let $\mathbb{E}_{G}:=\left(\left[s^{*} E_{G} \rightrightarrows E_{G}\right], \widetilde{s}, \widetilde{t}, \widetilde{m}, \widetilde{e}, \widetilde{i}\right)$ be a principal $G$-bundle over $\mathbb{X}$. We will show that $\mathbb{E}_{G}=\left[s^{*} E_{G} \rightrightarrows E_{G}\right]$ has a connection $\widetilde{\mathscr{H}}$ induced by $\mathscr{H}$. Let

$$
\phi_{X_{1}}: s^{*} E_{G} \rightarrow X_{1}
$$

be the natural projection and let $d \phi_{X_{1}}: T s^{*} E_{G} \rightarrow T X_{1}$ be its differential. Define

$$
\widetilde{\mathscr{H}}:=\left(d \phi_{X_{1}}\right)^{-1}(\mathscr{H}) \subset T s^{*} E_{G} .
$$

It is straightforward to check that $\widetilde{\mathcal{H}}$ is a connection on the groupoid $\mathbb{E}_{G}=\left[s^{*} E_{G} \rightrightarrows E_{G}\right]$.

As noted above, using $\widetilde{\mathscr{H}}$, the tangent bundle $T E_{G}$ is turned into a vector bundle over $\mathbb{X}$. We recall that $\operatorname{At}\left(E_{G}\right)=\left(\alpha_{*} T E_{G}\right)^{G}$. Using the action of $G$, the vector bundle $T E_{G}$ over $\mathbb{E}_{G}=\left[s^{*} E_{G} \rightrightarrows E_{G}\right]$ descends to make $\operatorname{At}\left(E_{G}\right)$ a vector bundle over $\mathbb{X}$. All the morphisms of the vector bundle $\operatorname{At}\left(E_{G}\right)$ over $\mathbb{X}$ are given by descent of the corresponding morphisms of the vector bundle $T E_{G}$ over $\mathbb{E}_{G}=\left[s^{*} E_{G} \rightrightarrows E_{G}\right]$.

Consequently, the exact sequence (3) of vector bundles over $X_{0}$ is an exact sequence of vector bundles over the groupoid $\mathbb{X}$.

Definition $4.1 A$ connection on a principal $G$-bundle $\mathbb{E}_{G}=\left[s^{*} E_{G} \rightrightarrows E_{G}\right]$ over a groupoid $\mathbb{X}=\left[X_{1} \rightrightarrows\right.$ $\left.X_{0}\right]$ is a splitting of the Atiyah sequence of vector bundles over $\mathbb{X}$ given by (3). It is said to be flat if it is integrable.

(Flat) connections behave again well with respect to pullbacks of cartesian morphisms and Morita equivalences of groupoids. So we have as observed before by Laurent-Gengoux, Stiénon and Xu [14] as well as by Tang [17] (see also [5]):

Proposition 4.1 If $\mathbb{X}$ and $\mathbb{Y}$ are Morita equivalent groupoids, then there is an equivalence between the respective categories of principal G-bundles with (flat) connections over $\mathbb{X}$ and $\mathbb{Y}$.

Therefore we can speak of (flat) connections for principal $G$-bundles on a stack $\mathfrak{X}$ (see [5]). In general these might not exist, but for principal $G$-bundles on orbifolds, i.e. proper étale Deligne-Mumford stacks, connections always exist (see also [15]).

A stack $\mathfrak{X}$ determines a collection of Morita equivalent groupoids associated to the presentations of the stack and any principal $G$-bundle $\mathfrak{E}_{G}$ on $\mathfrak{X}$ is given by principal $G$-bundles $E_{G} \rightarrow X_{0}$ over each atlas $X_{0} \rightarrow \mathfrak{X}$ satisfying appropriate glueing conditions (see for example [3]). We have the following [5]:

Theorem 4.2 Giving a (flat) connection for a principal $G$-bundle $\mathfrak{E}_{G}$ over a stack $\mathfrak{X}$ is equivalent to giving (flat) connections for the above principal $G$-bundles on the associated groupoids which are compatible with pullbacks along cartesian morphisms. Giving a (flat) connection for a principal G-bundle $\mathbb{E}_{G}$ on a groupoid $\mathbb{X}$ gives a (flat) connection for the principal $G$-bundle $\mathfrak{B E}_{G}$ of $\mathbb{E}_{G}$-torsors over the classifying stack $\mathfrak{B X}$.

\section{Curvature and characteristic differential forms}

Let $\mathscr{H}$ be a connection on the groupoid $\mathbb{X}=\left[X_{1} \rightrightarrows X_{0}\right]$. Using the canonical decomposition of the tangent space

we get a projection

$$
T X_{1}=\mathscr{H} \oplus \operatorname{kernel}(d s)
$$

$$
\wedge^{j} T X_{1} \rightarrow \wedge^{j} \mathscr{H} \hookrightarrow \wedge^{j} T X_{1} .
$$

The composition $\wedge^{j} T X_{1} \rightarrow \wedge^{j} T X_{1}$ gives by duality an endomorphism of the space of $j$-forms on $X_{1}$. 
For a differential form $\omega$ on $X_{1}$, the differential form on $X_{1}$ induced by the above endomorphism will be denoted by $H(\omega)$.

Definition $5.1 A$ differential $j$-form on the groupoid $\left[X_{1} \rightrightarrows X_{0}\right]$ is a differential $j$-form $\omega$ on $X_{0}$ such that $H\left(s^{*} \omega\right)=H\left(t^{*} \omega\right)$.

We have the following basic property:

Lemma 5.2 Assume that the distribution $\mathscr{H} \subset T X_{1}$ is integrable. Let $\omega$ be a differential form on $X_{0}$ such that $H\left(s^{*} \omega\right)=H\left(t^{*} \omega\right)$. Then $H\left(s^{*} d \omega\right)=H\left(t^{*} d \omega\right)$.

The space of connections on a principal $G$-bundle $E_{G}$ over the groupoid $\mathbb{X}$ is an affine space for the space of all $\operatorname{ad}\left(E_{G}\right)$-valued 1-forms on the groupoid [5].

Henceforth, we will assume that the distribution $\mathscr{H} \subset T X_{1}$ is integrable, i.e., a flat connection.

Consider the Atiyah sequence in (3). The Lie bracket of vector fields defines a Lie algebra structure on the sheaves of sections of all three vector bundles. The Lie algebra structure on the sheaf of sections of $\operatorname{ad}\left(E_{G}\right)$ is linear with respect to the multiplication by functions on $X_{0}$, or in other words, the fibers of $\operatorname{ad}\left(E_{G}\right)$ are Lie algebras.

The Lie algebra of $G$ will be denoted by $\mathfrak{g}$. Recall that $\operatorname{ad}\left(E_{G}\right)=\left(E_{G} \times \mathfrak{g}\right) / G$ is given by the adjoint action of $G$ on $\mathfrak{g}$. Since this adjoint action of $G$ preserves the Lie algebra structure of $\mathfrak{g}$, it follows that the fibers of $\operatorname{ad}\left(E_{G}\right)$ are Lie algebras identified with $\mathfrak{g}$ up to conjugations.

Given any splitting of the Atiyah sequence (3)

$$
D: T X_{0} \rightarrow \operatorname{At}\left(E_{G}\right)
$$

the obstruction for $D$ to be compatible with the Lie algebra structure is given by a section

$$
K(D) \in H^{0}\left(X_{0}, \operatorname{ad}\left(E_{G}\right) \otimes \Omega_{X_{0}}^{2}\right),
$$

which is an $\operatorname{ad}\left(E_{G}\right)$-valued 2-form on the groupoid $\mathbb{X}$. The section $K(D)$ is called the curvature of the connection $D$ on the principal $G$-bundle $\mathbb{E}_{G}=\left[s^{*} E_{G} \rightrightarrows E_{G}\right]$ over the groupoid $\mathbb{X}=\left[X_{1} \rightrightarrows X_{0}\right]$. The following result allows for a general Chern-Weil theory for constructing characteristic classes [5], which in the case of étale groupoids was developed before in [9] and [15], but where the characteristic classes are living in different cohomological spaces tailored directly for leaf spaces of foliations and étale groupoids.

Theorem 5.3 For any invariant form $\nu \in\left(\operatorname{Sym}^{k}(\mathfrak{g})\right)^{G}$, the closed $2 k$ form $\nu(K(D))$ on $X_{0}$ is a form on the groupoid.

\section{Acknowledgements}

We are very grateful to the referee for suggesting several improvements. The second author would like to thank the Tata Institute of Fundamental Research in Mumbai and the University of Leicester for financial support. The first author acknowledges the support of the J. C. Bose fellowship.

\section{References}

[1] M. F. Atiyah, Complex analytic connections in fibre bundles, Trans. Amer. Math. Soc. 85 (1957), 181-207.

[2] K. Behrend, On the de Rham cohomology of differential and algebraic stacks, Adv. Math. 198 (2005), 583-622.

[3] K. Behrend, P. Xu, Differentiable Stacks and Gerbes, Jour. Symp. Geom. Vol. 9 (2011), 285-341.

[4] I. Biswas, S. Majumder, M. L. Wong, Root stacks, principal bundles and connections, Bull. Sci. Math. 136 (2012), 369-398. 
[5] I. Biswas, F. Neumann, Atiyah sequences, connections and Chern-Weil theory for algebraic and differentiable stacks, in preparation.

[6] R. Bott, H. Shulman, J. Stasheff, On the de Rham theory of certain classifying spaces, Adv. Math. 20 (1976), 43-56.

[7] J. Cheeger, J. Simons, Differential characters and geometric invariants, Geometry and topology (College Park, Md., 1983/84), 50-80, Lecture Notes in Mathematics, Vol. 1167, Springer-Verlag, Berlin-New York, 1985.

[8] M. Crainic, R. J. Fernandes, Secondary characteristic classes of Lie algebroids, Quantum field theory and noncommutative geometry, 157-176, Lecture Notes in Phys. 662, Springer, Berlin, 2005.

[9] M. Crainic, I. Moerdijk, Čech-De Rham theory for leaf spaces of foliations, Math. Ann. 328 (2004), 59-85.

[10] H. Esnault, Algebraic differential characters. Regulators in analysis, geometry and number theory, 89-115, Progr. Math., 171, Birkhüser Boston, 2000.

[11] M. Felisatti, F. Neumann, Secondary theories for étale groupoids, Contemp. Math. Vol. 571 (2012), 135-151.

[12] J. Heinloth, Notes on differentiable stacks, Mathematisches Institut Seminars 2004-2005, (Y. Tschinkel ed.), GeorgAugust Universität Göttingen, 1-32.

[13] E. Lerman, A. Malkin, Hamiltonian group actions on symplectic Deligne-Mumford stacks and toric orbifolds, Adv. Math. 229 (2012), 984-1000.

[14] C. Laurent-Gengoux, P. Stiénon, P. Xu, Non-abelian differentiable gerbes, Adv. Math. 220 (2009), 1357-1427.

[15] C. Laurent-Gengoux, J.-L. Tu, P. Xu, Chern-Weil map for principal bundles over groupoids, Math. Zeit. 255 (2007), 451-491.

[16] M. A. Salazar Pinzón, Pfaffian groupoids, PhD thesis, Utrecht 2013, arXiv:1306.1164v2 [math.DG].

[17] X. Tang, Deformation quantization of pseudo-symplectic (Poisson) groupoids, Geom. Funct. Anal. 16 (2006), 731-766. 